\title{
Angular anisotropy of intermediate energy nucleon-induced fission of actinide and subactinide nuclei
}

\author{
V.P. Eismont ${ }^{1, \text { a }}$, N.P. Filatov ${ }^{1}$, A.N. Smirnov ${ }^{1}$, and J. Blomgren ${ }^{2}$ \\ 1 V.G. Khlopin Radium Institute, 2oi Murinskiy Prospect 28, Saint-Petersburg 194021, Russia \\ 2 Department of Neutron Research, Uppsala University, Box 525, 75120 Uppsala, Sweden
}

\begin{abstract}
New results of measurements of the angular distributions of proton-induced fission fragments of ${ }^{182,183,184,186} \mathrm{~W}$ in the proton energy region $50-180 \mathrm{MeV}$ are presented. The results are discussed together with earlier data, obtained for subactinide nuclei ${ }^{204,206,207,208} \mathrm{~Pb}$ and ${ }^{209} \mathrm{Bi}$ and actinide nuclei ${ }^{232} \mathrm{Th}$ and ${ }^{233,235,238} \mathrm{U}$, for both protonand neutron-induced fission. Similarity is noted of values and energy dependences of angular anisotropy of fission induced by protons and neutrons (taking into account a trivial effect of Coulomb factor) as well as influence on the anisotropy value of the emission character of fission of actinide nuclei and its connection with parameter of fissionability $Z^{2} / A$.
\end{abstract}

\section{Introduction}

Studies of fission fragment angular anisotropies is a way to determine the state of a fissioning nucleus at the saddle point shape, angular momentum and temperature. For the understanding of dynamics of fission process the knowledge of these key characteristics is necessary in wide range of fissioning nuclei, their excitation energies and angular momenta. With this goal, in framework of ISTC projects measurements have carried out of the angular anisotropies in neutron-induced fission of ${ }^{232} \mathrm{Th}$ and ${ }^{238} \mathrm{U}$ in the $20-160 \mathrm{MeV}$ energy region and ${ }^{209} \mathrm{Bi}$ at $75 \mathrm{MeV}$ [1], and in proton-induced fission of 204,206,207,208 $\mathrm{Pb}$ and ${ }^{209} \mathrm{Bi}$ in the 50-180 MeV energy region [2]. The results have been analyzed together with compiled data on angular anisotropies in proton-induced fission of ${ }^{232} \mathrm{Th}$ and ${ }^{233,235,238} \mathrm{U}$ [3] and subactinides [4]. Analysis of the experimental data for actinides have shown that the value of the anisotropy depends on ratio of neutron and fission widths, $\Gamma_{n} / \Gamma_{f}$, of fissioning nuclei which determine the multi-chance structure of fission of these nuclei (its dependence on the fissionability of nucleus).

In the present work new results of measurements of the angular distributions of proton-induced fission fragments of $182,183,184,186 \mathrm{~W}$ in the energy range $50-180 \mathrm{MeV}$ are presented and compared with compiled data for adjacent nuclei, ${ }^{185} \mathrm{Re}$ and ${ }^{181} \mathrm{Ta}$, and for subactinides in lead-bismuth region. The discussion is carried out in frame of the standard statistical (transition state) model taking into account the characteristics of the intermediate compound nuclei formed in process of interaction of nucleons with target nuclei.

\section{Experimental results}

Fission fragment angular distributions for intermediate energy proton-induced fission of ${ }^{182,183,184,186} \mathrm{~W}$ have been measured at the proton beam of the Gustaf Verner cyclotron of the The Svedberg Laboratory of the Uppsala University, Sweden. The

\footnotetext{
${ }^{a}$ Presenting author, e-mail: 105@atom.nw.ru
}

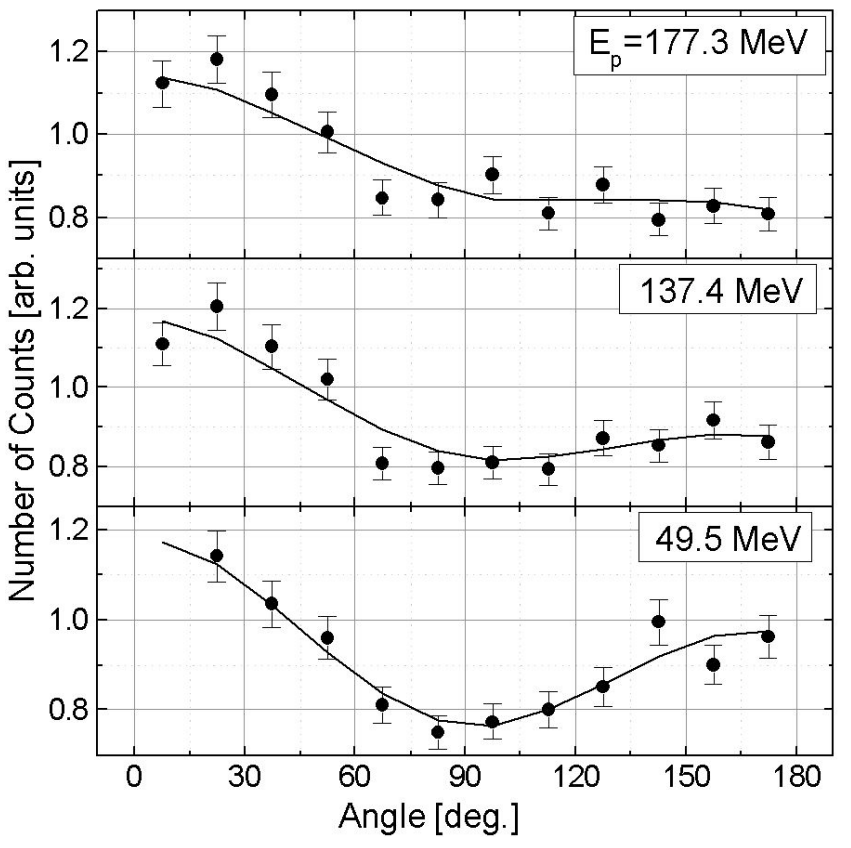

Fig. 1. Angular distributions of the ${ }^{184} \mathrm{~W}$ fission fragments at the proton energies $49.5,137.4$, and $177.3 \mathrm{MeV}$ in the laboratory frame. Curves are the model calculations.

measurements were carried out using a vacuum chamber with thin-film breakdown counters (TFBC) for fission fragment detection placed in the angular ranges of about $0^{\circ}-90^{\circ}$ and $90^{\circ}-180^{\circ}$ relatively to the proton beam direction. The targets were prepared from enriched mixtures of tungsten isotopes ${ }^{182} \mathrm{~W}(99.1 \%),{ }^{183} \mathrm{~W}(99.8 \%),{ }^{184} \mathrm{~W}(99.2 \%)$ and ${ }^{186} \mathrm{~W}(99.9 \%)$ in the $\mathrm{WO}_{3}$ chemical form. The target layer thicknesses were about $2 \mathrm{mg} / \mathrm{cm}^{2}$. Measurements were carried out at proton energies 49.5, 137.4 and 177.3 MeV. Experimental angular distributions obtained were fitted by computer code based on standard kinematics expressions for nuclear reactions as well as parameters of fission targets, mass and energy distributions of fission fragments and detection properties of the TFBC. 
Table 1. Anisotropy factor for proton-induced fission fragments.

\begin{tabular}{llcl}
\hline Isotope & \multicolumn{3}{c}{ Proton energy, MeV } \\
\cline { 2 - 4 } & $49.5 \pm 0.1$ & $137.4 \pm 1.0$ & $177.3 \pm 1.2$ \\
\hline${ }^{186} \mathrm{~W}$ & $0.30 \pm 0.06$ & $0.25 \pm 0.05$ & $0.14 \pm 0.05$ \\
${ }^{18} \mathrm{~W}$ & $0.40 \pm 0.06$ & $0.25 \pm 0.07$ & $0.14 \pm 0.05$ \\
${ }^{183} \mathrm{~W}$ & $0.40 \pm 0.06$ & - & $0.14 \pm 0.03$ \\
${ }^{182} \mathrm{~W}$ & $0.40 \pm 0.06$ & $0.25 \pm 0.12$ & $0.14 \pm 0.03$ \\
\hline
\end{tabular}

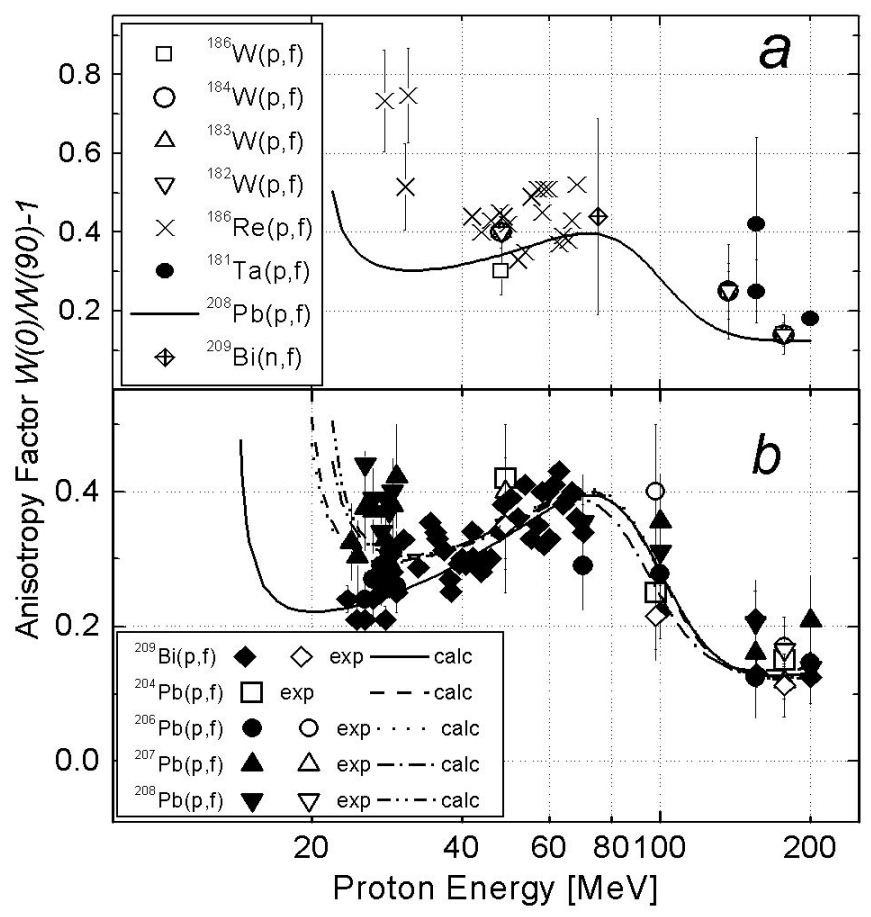

Fig. 2. Energy dependence of the anisotropy factor for protoninduced fission.

The experimental setup, measurement conditions and procedure of process of experimental results are described in details in our reports [2,5].

In figure 1 the experimental angular distributions of the ${ }^{184} \mathrm{~W}$ fission fragments in the laboratory frame are presented for three energies of incident protons. The anisotropy factors, $\mathrm{C}=\mathrm{W}^{\prime}\left(0^{\circ}\right) / \mathrm{W}^{\prime}\left(90^{\circ}\right)-1$, for ${ }^{182,183,184,186} \mathrm{~W}$ are given in table 1 and shown in figure $2 \mathrm{a}$ together with compiled data for adjacent nuclei, ${ }^{185} \mathrm{Re}$ and ${ }^{181} \mathrm{Ta}[6]$.

\section{Angular anisotropy for subactinides}

In our previous study [4] we have performed semi-empirical analysis of energy dependence of angular anisotropy for subactinide nuclei in the lead-bismuth region. Experimental data on proton-induced fission for separated lead isotopes and bismuth were taken from compilation [6] and supplemented by our measurements [2]. The analysis has been carried out in the frame of transition state model [7], where the anisotropy factor $C$ is merely connected with the mean square of the angular momentum of a nucleus at the saddle point, $\left\langle I^{2}\right\rangle$, and the dispersion of the momentum projection on the axis of fissioning nucleus, $K_{o}^{2}: C=\left\langle I^{2}\right\rangle / K_{0}^{2}$, where $K_{o}^{2}=\left(I / \hbar^{2}\right) J_{\text {eff }} T$, where $J_{\text {eff }}$ is the effective moment of inertia, and $T$ is the temperature of a nucleus in this state. Because of complexity of quantitative consideration of cascade and pre-equilibrium particle emission resulting in formation of a broad set of residual nuclei, for subacinide region we applied an "integral" description where $\left\langle l^{2}\right\rangle$ and $\tilde{E}_{0}^{2}$ are averaged over the states of fissioning nuclei. The results of these calculations are summarized in figure $2 b$.

It is seen from figure 2 that experimental data sets for leadbismuth and tungsten regions are very similar. In particular, despite of considerable widening of range of average parameter $Z^{2} / A$ of composite system (incident particle + target nucleus) relatively to ref. [4] - from 33.60 (for ${ }^{209} \mathrm{Bi}+\mathrm{p}$ ) and 32.96 (for ${ }^{208} \mathrm{~Pb}+\mathrm{p}$ ) to 30.08 (for ${ }^{186} \mathrm{~W}+\mathrm{p}$ ) - the maximal values of the anisotropy factor practically did not change. This testifies about independence $K_{o}^{2} \sim J_{\text {eff }} T$, and probably $I$ (as far as approximate constancy of $J_{e f f}$ for these nuclei is predicted by liquid drop model [8]) on $Z^{2} / A$ for subactinide nuclei having weak fissionability and accordingly low probability of emission fission. Thus the calculated energy dependences of the anisotropy factors for lead isotopes can be used for all tungsten isotopes. In figure $2 \mathrm{a}$ the curve calculated for ${ }^{208} \mathrm{~Pb}$ is used as for nucleus with closest fission barrier. It is seen from figure 2 that the calculated curve satisfactorily describe experimental results.

Measurements of angular anisotropy in neutron-induced fission in intermediate energy region have been started in ref. [1]. One of the first results was measurement of angular distribution of fission fragments and anisotropy factor for the ${ }^{209} \mathrm{Bi}(\mathrm{n}, \mathrm{f})$ reaction at neutron energy $75 \mathrm{MeV}$ using a Frish gridded ionization chamber [9].

A physical base for comparison of data obtained at neutrons and protons can be found in hypothesis of quasicompound nucleus formed in interaction of heavy nuclei with intermediate energy particles [10]. The "compound nucleus" effect is manifested in similarity of number of observed characteristics for composite systems with the same charge $Z$, mass $A$ and excitation energy $E^{*}$, independently on incident particle (proton or neutron), but taking into account trivial factor of Coulomb barrier for protons. This effect, probably, reflects closeness of parameters of interim compound nuclei formed as a result of intra-nuclear cascade and preliminary emission. For two reactions ${ }^{208} \mathrm{~Pb}+\mathrm{p}$ and ${ }^{209} \mathrm{Bi}+\mathrm{n}$ the average values $\langle Z\rangle$, $\langle A\rangle$ and $\left\langle E^{*}\right\rangle$, versus the incident nucleon energy as well as the shapes of their distributions for three values of the projectile energy are shown in figure 3 . The data are extracted from calculations by CEM03.01 event generator [11]. Similarity of average values of the above mentioned parameters have been established earlier in ref. [12] as results of calculations by the TALYS code [13].

In case of angular anisotropy account of Coulomb barrier results in change of introduced angular momentum, thus it appears that $C_{n}=(E /(E-V)) C_{p}$. Experimental value of $C_{n}$ for ${ }^{209} \mathrm{Bi}(\mathrm{n}, \mathrm{f})-0.52 \pm 0.20$ corrected to this factor is compared with $C_{p}$ for the ${ }^{208} \mathrm{~Pb}(\mathrm{p}, \mathrm{f})$ in figure $2 \mathrm{a}$. It is seen that the values do not contradict each other although they have large uncertainties. 

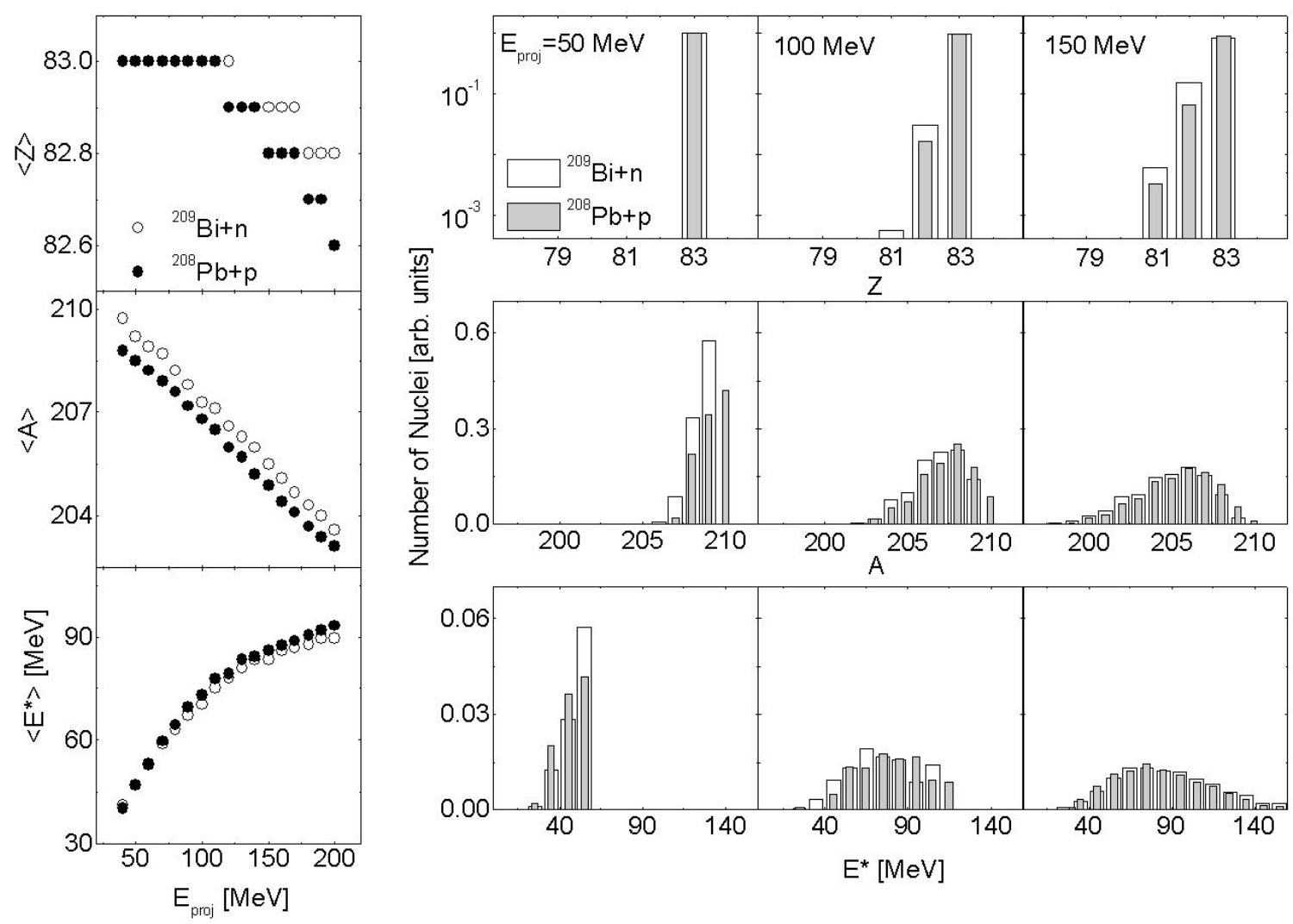

Fig. 3. Average charge, mass and excitation energy and their distributions vs projectile energy for reactions ${ }^{209} \mathrm{Bi}+\mathrm{n}$ and ${ }^{208} \mathrm{~Pb}+\mathrm{p}$ calculated by the code CEM03.

\section{Angular anisotropy for actinides}

Measurements of angular anisotropies for neutron-induced fission of ${ }^{232} \mathrm{Th}$ and ${ }^{238} \mathrm{U}$ in the neutron energy region 20 $160 \mathrm{MeV}[1,14]$ have contributed in more detailed comparison of values and energy dependences of the anisotropy for protons and neutrons as well as in determination of physical factors affecting the anisotropy for actinide nuclei. In ref. [3] all compiled experimental data on angular anisotropies for proton-induced fission of ${ }^{232} \mathrm{Th},{ }^{238} \mathrm{U},{ }^{233} \mathrm{U}$ and ${ }^{235} \mathrm{U}$ have been analyzed. The best agreement of calculated in frame of transition state model (like for subactinide nuclei) and experimental values of anisotropy factors has been obtained for excitation energy at a saddle point for ${ }^{238} \mathrm{U}$ approximately to about $8 \mathrm{MeV}$ more than for ${ }^{232} \mathrm{Th}$ that has been connected with difference in fissionability for these nuclei.

The dependence of anisotropy on the fissility parameter $Z^{2} / A$ in fission induced by low-energy neutrons but at energies higher than the threshold of the emission fission was discussed in refs. $[15,16]$. It is interesting that parameter $Z^{2} / A$ of the composite systems plays the same role at higher energies when the mechanism of the reaction becomes more complex and the excitation energy of fissioning nuclei becomes higher. It has been assumed that, as in the case of subactinide nuclei, the anisotropy factor for actinides in neutron-induced fission can be calculated using the anisotropy factor for the same energy protons and the composite system with the same parameter $Z^{2} / A$, taking into account the difference of introduced momenta: $C n=(E /(E-V)) C p$. In our case the energy dependence of anisotropy for the reaction ${ }^{238} \mathrm{U}+\mathrm{n}\left(Z^{2} / \mathrm{A}=\right.$ $\left.92^{2} / 239=35.41\right)$ was calculated using the reaction ${ }^{232} \mathrm{Th}+$ $\mathrm{p}\left(\mathrm{Z}^{2} / \mathrm{A}=91^{2} / 233=35.54\right)$. The positive result of this approach for converting data for protons to neutron ones has made it possible to apply the reverse procedure to the case of the ${ }^{232} \mathrm{Th}(\mathrm{n}, \mathrm{f})$ reaction and thus to obtain the data on the anisotropy for the hypothetical case of proton fission of the composite nucleus with $Z^{2} / A=34.76$. Thus the use of neutron data allowed to expanding the analyzed range of fissilities.

Data on anisotropy factors for $40 \mathrm{MeV}$ proton-induced fission (including converted from data on neutron-induced ones) and all known data on anisotropy of fission induced by deuterons and alpha-particles have been compared with data on ratio of neutron and fission widths, $\Gamma_{n} / \Gamma_{f}$ (determining fissionability of nuclei $P_{f}=\left(1+\Gamma_{n} / \Gamma_{f}\right)^{-1}$ in the range of parameter of fissionability $Z^{2} / A$ about $35 \div 38$ (see fig. 4 ). It has been concluded that similarity of behavior of the anisotropy and the probability of neutron emission before the saddle point which is determined by $\Gamma_{n} / \Gamma_{f}$ shows that the temperature at the saddle point remains to be an important factor influencing the anisotropy of fission heavy nuclei induced by intermediate energy nucleons as at low energies.

\section{Conclusion}

Experimental studies of angular anisotropies of intermediate energy nucleon-induced fission in the wide range of parameter 


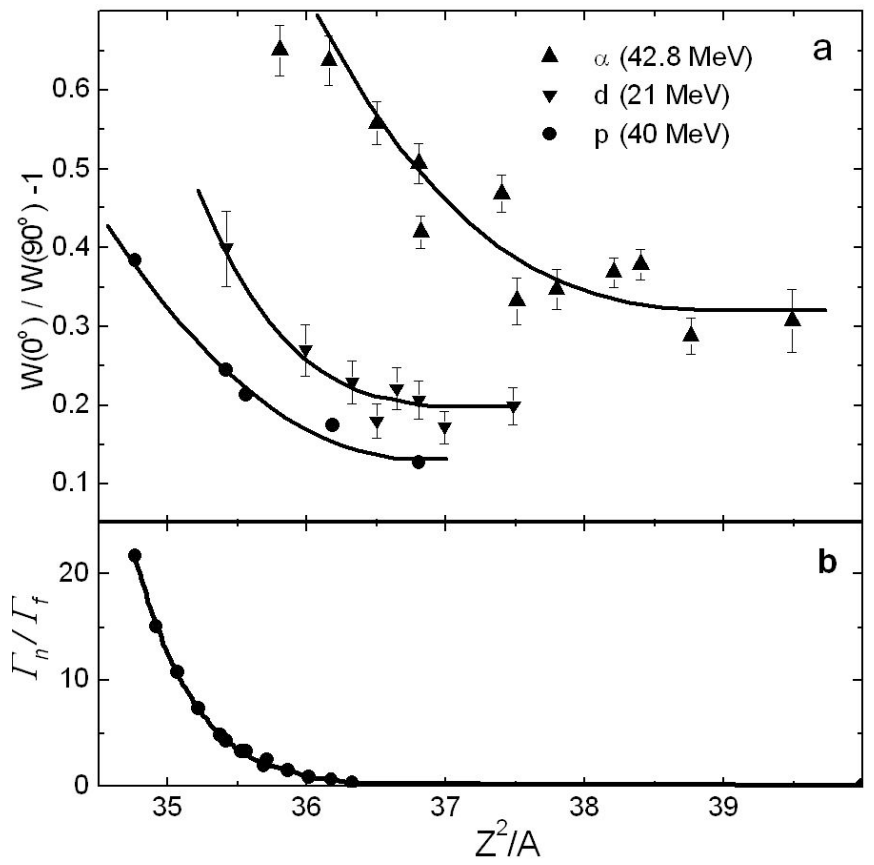

Fig. 4. The dependence of anisotropy coefficient for fission induced by $\mathrm{p}, \mathrm{d}$ and $\alpha$-particles (the experimental data on $\mathrm{d}$ and $\alpha$-particles are from the ref. [17], the curves are ours) on composite nucleus fissility parameter (a), the dependence of the ratio of neutron and fission widths at $\mathrm{E}^{*} \approx 10 \mathrm{MeV}$ on composite nucleus fissility parameter (b).

of fissionability of nuclei has shown a major similarity of values and energy dependencies of the anisotropy for protons and neutrons. Observed difference in the projectile energy region below $50-60 \mathrm{MeV}$ is connected with effect of Coulomb factor diminishing the angular momentum introduced by protons and reducing the anisotropy factor. It reflects the effect of "compound nucleus" being manifested in similarity of characteristics of fission reaction for composite systems of equal nucleon composition and excitation energy. Such similarity is seen from calculations of parameters of interim compound nuclei carried out in frame of modern model codes CEM03 and TALYS. At the same time a significant difference takes place between actinides and subactinides in dependence of the anisotropy of fission by both charged particles and neutrons on parameter $\mathrm{Z}^{2} / \mathrm{A}$. The value of the anisotropy coefficient for subactinides practically does not depend on this parameter, while for actinides it drops strongly with the parameter increase. It is connected with strong dependence of fissionability on $\mathrm{Z}^{2} / \mathrm{A}$ resulting in difference of excitation energies in saddle point, determining the value of anisotropy in transition state model.

The authors are thankful to Dr A.V. Prokofiev for active participation in the experimental part of the study and Dr S.G. Mashnik for providing model calculation by the CEM03.01 code. This work was performed in framework of the ISTC project 2213.

\section{References}

1. V.P. Eismont (Project Manager), Final Project Technical Report of ISTC 540-97, V.G. Khlopin Radium Institute, St.-Petersburg, 1999.

2. V.P. Eismont (Project Manager), Final Project Technical Report of ISTC 1309-99, V.G. Khlopin Radium Institute, St.-Petersburg, 2002.

3. V.P. Eismont et al., J. Nucl. Sci. Technol., Suppl. 2, 299 (2002).

4. V.P. Eismont et al., in Proc. Int. Conf. on Nuclear Data for Science and Technology, Santa Fe, NM, Sept. 26-Oct. 1, 2004, AIP Conf. Proc. 769 (2005), p. 633.

5. V.P. Eismont (Project Manager), Final Project Technical Report of ISTC 2213-03, V.G. Khlopin Radium Institute, St.-Petersburg, 2006.

6. A. I. Obukhov, Phys. Part. Nuclei 32, 162 (2001).

7. H. Halpern, V.M. Strutinskiy, Proc. of the Second United Nations Int. Conf. on Peaceful Uses of Atomic Energy, Geneva, Switzerland, 1958 (United Nations, Geneva, Switzerland, 1958), p. 408; R. Vandenbosch, R. Huizenga, Nuclear Fission (Academic Press, New York, 1973).

8. V.M. Strutinskiy, Sov. J. Nucl. Phys. 1, 821 (1965) (in Russian).

9. V.P. Eismont et al., in Proc. Int. Conf. on Nuclear Data for Science and Technology, May 19-24, 1997, Trieste, Italy, p. 658

10. A.N. Smirnov et al. (these proceedings).

11. S.G. Mashnik et al., LANL Report LA-UR-05-2686, Los Alamos, 2005; E-prints: nucl-th/0502019; nucl-th/0503061.

12. A.N. Smirnov et al., in Proc. Int. Conf. on Nuclear Data for Science and Technology, Santa Fe, NM, Sept. 26-Oct. 1, 2004, AIP Conf. Proc. 769 (2005), p. 780.

13. A.J. Koning et al., in Proc. Int. Conf. on Nuclear Data for Science and Technology, Santa Fe, NM, Sept. 26 - Oct. 1, 2004, AIP Conf. Proc. 769 (2005), p. 1154.

14. G.A. Tutin et al., Nucl. Instrum. Meth. Phys. Res. A 457, 646 (2001).

15. R.L. Henkel, J.E. Brolley, Phys. Rev. 103, 1292 (1956).

16. A.N. Protopopov, V.P. Eismont, JETP 34, 250 (1958) (in Russian).

17. T. Datta et al., Z. Phys. A 351, 305 (1995). 